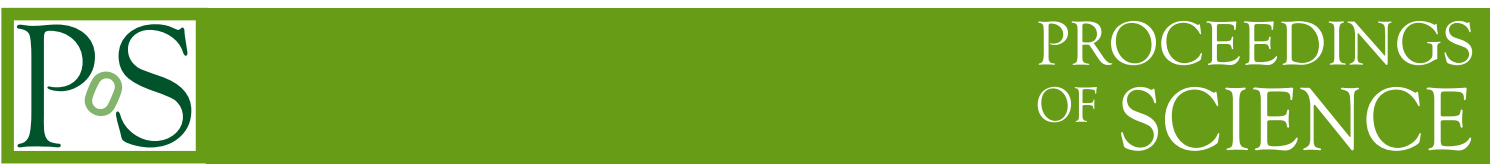

\title{
Exclusive production at LHC
}

\author{
Nicolas Schul*, for the CMS collaboration \\ Center for Particle Physics and Phenomenology (CP3) \\ Université catholique de Louvain \\ Louvain-la-Neuve, Belgium \\ E-mail: nicolas.schul@uclouvain.be
}

\begin{abstract}
The exclusive production of dimuons, $p p \rightarrow p \mu^{+} \mu^{-} p$, is studied in a Monte Carlo simulation for $p p$ collisions at $7 \mathrm{TeV}$ and $14 \mathrm{TeV}$. First, a continuum signal due to the QED process $\gamma \gamma \rightarrow$ $\mu^{+} \mu$ is discussed, and then in the mass region $8<m\left(\mu^{+} \mu\right)<12 \mathrm{GeV}$, search for the exclusive photoproduction of $\Upsilon$ mesons is described. Outlook for the studies of the exclusive processes with forward proton detectors are also presented.
\end{abstract}

XVIII International Workshop on Deep-Inelastic Scattering and Related Subjects April 19 -23, 2010

Convitto della Calza, Firenze, Italy

\footnotetext{
* Speaker.
} 


\section{Introduction}

At hadron colliders, exclusive production refers to processes where both protons remain intact after the interaction and may escape undetected along the beamline. These events result in clean and unusual experimental signature including only a few particles registered by the central detector, no hadronic activity in the forward region, and the presence of two scattered protons. That kind of interactions may arise from three different physical processes: two-photon production $(\gamma \gamma)$, elastic photo-production $(\gamma \mathbb{P})$ and central exclusive production (CEP).

In the first case, early studies of final states exclusively produced in the two-photon fusion will concentrate on dimuon, dielectron and $W^{+} W^{-}$pairs. At higher luminosities, sufficient event rates will allow to search for physics beyond the Standard Model such as exclusive production of supersymmetric pairs $\left(\tilde{\ell}^{+} \tilde{\ell}^{-}, \tilde{\chi}_{1}^{+} \tilde{\chi}_{1}^{-}, H^{+} H^{-}\right)[1,2]$. In addition, studies of events produced by $\gamma \mathbb{P}$ interactions include the exclusive production of the $Z$ boson and vector mesons - although only the heaviest $\Upsilon$ state will be detectable, due to a strongly decreasing detector efficiency for low $p_{T}$ particles from the quarkonia decays. Finally, a large variety of final states resulting from CEP is under study, among them di-jets, $\chi_{b}, \gamma \gamma$ and Higgs boson [3, 4].

In the context of the current LHC schedule, two kinds of analyzes may be considered. During the LHC early running, with low event pile-up, studies of the exclusive events can be performed using only the main CMS detectors. As for example, a Monte Carlo study of the exclusive dimuon pairs is discussed in Sections 3 to 5 and in detail in [5]. In longer term, CMS collaboration is studying use of dedicated forward proton spectrometers, as it is presented in Section 6. In that case, detection of the two scattered protons help to a constrain of the kinematic and significantly reduce backgrounds.

\section{The CMS detector}

The central feature of the Compact Muon Solenoid (CMS) apparatus is a superconducting solenoid of $6 \mathrm{~m}$ internal diameter. Within the field volume are the silicon pixel and strip tracker, the crystal electromagnetic calorimeter (ECAL) and the brass/scintillator hadron calorimeter (HCAL). Muons are measured in gas-ionization detectors embedded in the steel return yoke. In addition to the barrel and endcap detectors, CMS has extensive forward calorimetry.

The inner tracker measures charged particles within the $|\eta|<2.5$ pseudorapidity range. It consists of silicon pixel and strip detector modules and is located in the $3.8 \mathrm{~T}$ field of the solenoid. The calorimeter cells are grouped in projective towers, of granularity $\Delta \eta \times \Delta \phi=0.087 \times 0.087$ at central rapidities and $0.175 \times 0.175$ at forward rapidities, and cover up to $|\eta|<5.2$. The muons are measured in the window $|\eta|<2.4$, with detection planes made of three technologies: Drift Tubes, Cathode Strip Chambers, and Resistive Plate Chambers. The very forward angles are covered by the CASTOR $(-5.3>\eta>-6.6)$ and Zero Degree $(|\eta|>8.3)$ calorimeters, made of quartz 
fibers/plates embedded in tungsten absorbers. A much more detailed description of CMS can be found elsewhere [6].

\section{Exclusive dimuon final states}

Exclusive dimuon events may arise from the three kinds of exclusive processes quoted above. Two-photon fusion of muon pairs is the main contribution and predictions from the LPAIR gen-
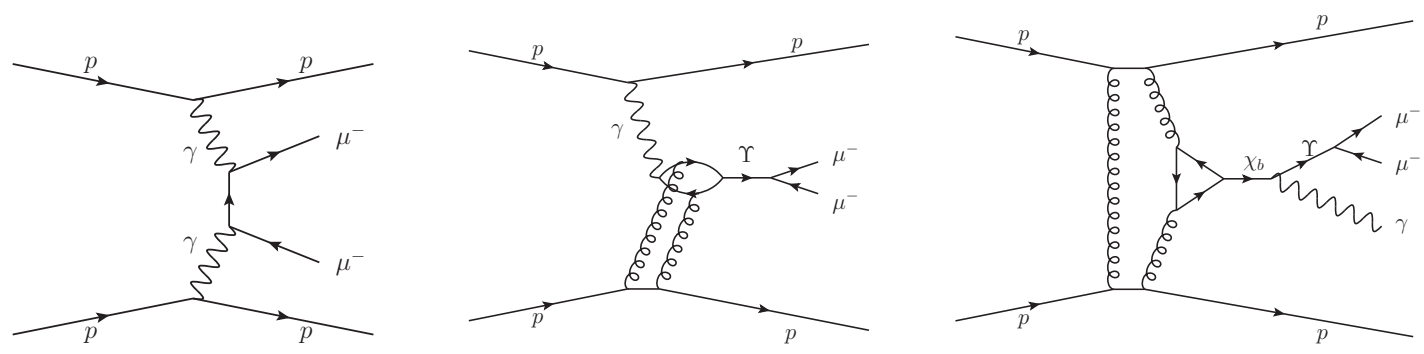

Figure 1: Feynman diagrams contributing to exclusive dimuons signal. Left: two-photon production of muon pair. Center: Upsilon photoproduction. Right: central exclusive production of $\chi_{b}$

erator [7] are displayed in Table 1 for 7 and $14 \mathrm{TeV}$ c.m.s. energies. It is worth notice that the cross-sections are very well known at the theoretical level, with $\mathscr{O}(1 \%)$, such that it is proposed to use these events to calibrate the integrated luminosity at the LHC. Moreover, it will provide a clean sample for the alignment of the future proton spectrometers [11].

\begin{tabular}{lc|cc}
\hline Production cross-section & & $7 \mathrm{TeV}$ & $14 \mathrm{TeV}$ \\
\hline Elastic $\gamma \gamma \rightarrow \mu^{-} \mu^{+}$ & $p_{T}^{\mu}>2 \mathrm{GeV},\left|\eta^{\mu}\right|<3$ & $72.6 \mathrm{pb}$ & $55.4 \mathrm{pb}$ \\
Inelastic $\gamma \gamma \rightarrow \mu^{-} \mu^{+}$ & $p_{T}^{\mu}>2 \mathrm{GeV},\left|\eta^{\mu}\right|<3$ & $90.6 \mathrm{pb}$ & $69.4 \mathrm{pb}$ \\
Elastic $\gamma p \rightarrow \Upsilon p \rightarrow \mu^{-} \mu^{+} p$ & $1 \mathrm{~S}, 2 \mathrm{~S}, 3 \mathrm{~S}$ & $62.0 \mathrm{pb}$ & $21.6 \mathrm{pb}$ \\
\hline
\end{tabular}

Table 1: Production cross-sections as given by the LPAIR and STARLIGHT generators, for a c.m.s. energy of 7 and $14 \mathrm{TeV}$. Elastic $\Upsilon$ photo-production value is the sum of the three resonances.

Exclusive $\Upsilon$ production resulting from the interaction (at leading order) of a pomeron with a $q \bar{q}$ excitation from a $\gamma^{*}$, can decay to two soft muons. The cross-section is expected to be related to the generalized gluon density of the proton as $\sigma \sim(g(x))^{2}$. The increasing gluon density at low- $x$ leads to a dependence of $\sigma \sim W^{1.7}$ at leading order in pQCD, where $W$ is the $\gamma p$ center of mass energy. Predictions from the STARLIGHT generator [8] are quoted in Table 1. Exclusive $\Upsilon$ sample will allow to test heavy-flavor diffraction at higher energies than previous measurements in $e p$ experiments [9]. Finally, CEP of $\chi_{b}$ decaying to one soft photon and one $\Upsilon$ may result in the same experimental final states if the photon goes undetected. As large uncertainties remain in the cross-section and the branching ratios determination, this contribution is neglected in the following. 


\section{Event selection}

While exclusive dimuon events from two-photon production or from $\Upsilon$ photo-production result from different physics processes, their experimental signatures are very similar. Therefore a common selection, based on the kinematics of the leptons and the lack of additional activity in the detector, can be used to select both types of events.

The main irreducible background for all processes arises from the 'inelastic' contribution when a photon emission is accompanied by a proton breaking. In a large fraction of these events, the proton remnant contents leave outside the central CMS coverage. In addition, other backgrounds taken into account include Drell-Yan production, quarkonium decays and heavy-flavor semi-leptonic decays. Both signal and background samples are passed to the full detector simulation, HLT emulation and reconstruction.

\subsection{Trigger and Kinematic selection}

Events are triggered if they pass the default low-luminosity Level 1 (L1) and High Level (HLT) dimuon triggers, which require two muons with $p_{T}>3 \mathrm{GeV}$. Further selection uses the highlyconstrained four-body final state to select events for which the transverse momentum balance satisfies the condition $\left|\Delta p_{T}\left(\mu^{+} \mu^{-}\right)\right|<2.0 \mathrm{GeV}$, and the muons are back-to-back in the transverse plane, i.e. acoplanarity satisfies $\left|\Delta \phi\left(\mu^{+} \mu^{-}\right)\right|>2.9$.

\subsection{Exclusivity selection}

In the no-pileup scenario assumed here, the exclusive signal is foreseen to have no calorimeter hits not associated with the muons, and no extra charged tracks attached to the $\mu^{+} \mu^{-}$vertex. The calorimeter exclusivity requirement is implemented by asking for events with no more than 5
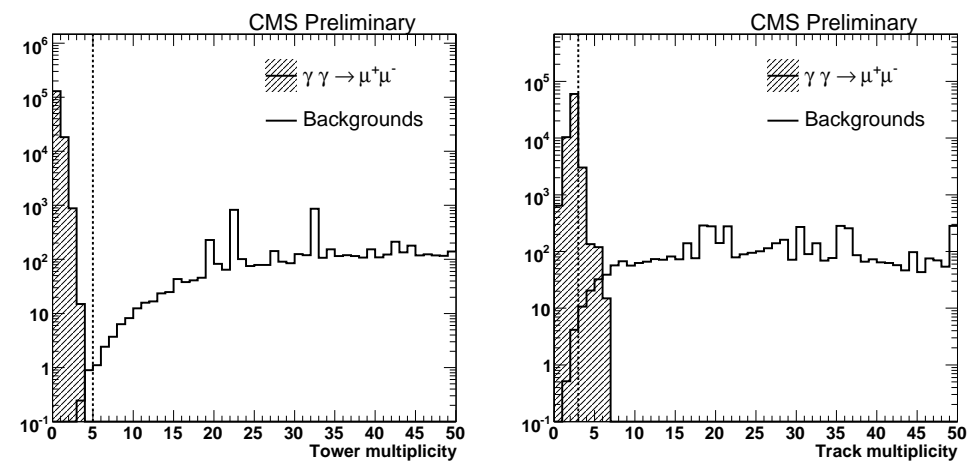

Figure 2: Distribution of the multiplicity of isolated calorimeter towers (left) and tracks (right) for muons for signal (shaded histogram) and (open histogram) backgrounds (arbitrary normalization). The dotted lines indicate the cut values.

calorimeter towers, separated from either muon candidates by $\Delta R>0.3$ in the $\eta-\phi$ plane, to be above the noise threshold of $5 \mathrm{GeV}$. The distributions of extra calorimeter towers and track multiplicities are shown in Figure 2 for signal and for backgrounds, in addition with the considered cuts. 
As inelastic processes have high efficiency to fulfill these exclusivity conditions, vetoing events with large activities in the CASTOR and/or ZDC forward detector will help to further suppress this irreducible background. Preliminary studies with Monte Carlo simulation show that approximatively $2 / 3$ of the inelastic events passing the exclusivity cuts quoted above will leave significant energy deposit in the forward coverage of the CMS.

\section{Results}

The dimuon invariant mass for $14 \mathrm{TeV}$ c.m.s. and after applying all trigger, kinematical and exclusivity selection plus forward calorimeters veto described above is shown in Figure 3 for an integrated luminosity of $100 \mathrm{pb}^{-1}$. The expected signal yields from the Monte Carlo exercice are:

$$
\begin{gathered}
N_{\text {Elastic }}\left(\gamma \gamma \rightarrow \mu^{+} \mu^{-}\right)=709 \pm 27, \\
N_{\text {Inelastic }}\left(\gamma \gamma \rightarrow \mu^{+} \mu^{-}\right)=223 \pm 15 \pm 42
\end{gathered}
$$

where the first uncertainty is the statistical error taken as $\sqrt{N}$ and the second corresponds to a $19 \%$ model-dependent uncertainty on the inelatic cross-section. Although the inelastic background remains significant, a statistical separation of the two contributions based on the reconstructed $|\Delta \phi|$ and $\left|\Delta p_{T}\right|$ would provide an integrated luminosity calibration with a $4 \%$ precision for $100 \mathrm{pb}^{-1}$.
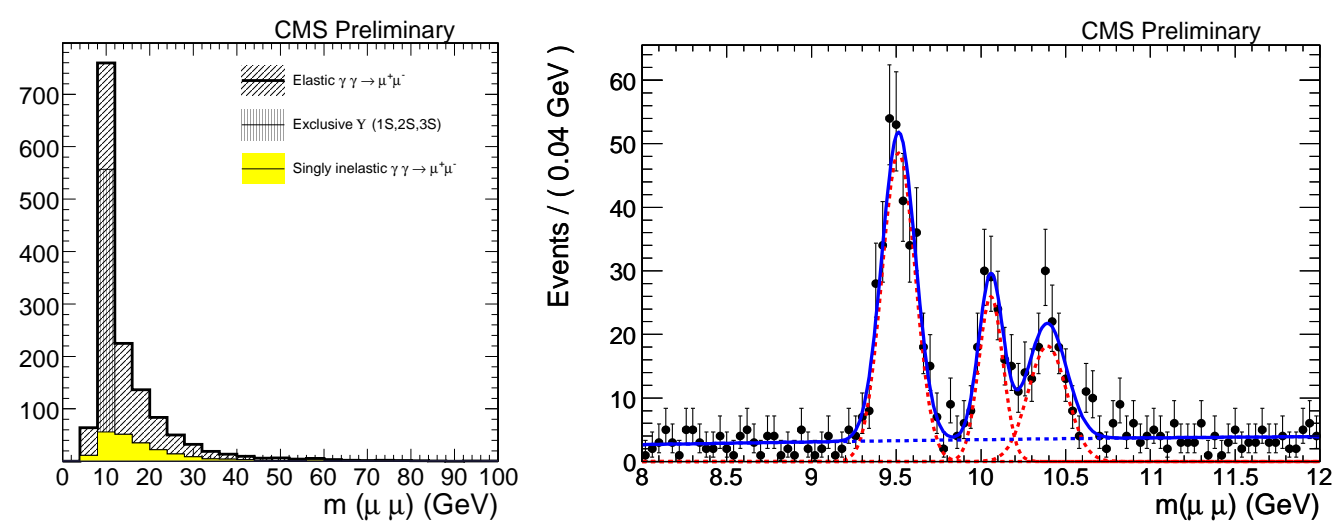

Figure 3: $\mu^{+} \mu^{-}$invariant mass for events passing the selection criteria, for an assumed luminosity of 100 $\mathrm{pb}^{-1}$. Left: all events. Right: $\Upsilon$ mass region. The dotted lines show the signal component due to the three $\Upsilon$ resonances, the dashed line shows the component due to the elastic and inelastic two-photon events, the solid line is the sum of the $\Upsilon$ and two-photon contributions.

The $\Upsilon$ photo-production signal can be extracted by performing a fit in the range $8<m<$ $12 \mathrm{GeV}$. The resonances $1 S, 2 S$ and $3 S$ are fitted with single gaussians, while the continuum from QED $\gamma \gamma \rightarrow \mu^{+} \mu^{-}$contribution is supposed to follow a second-order polynomial function. For an integrated luminosity of $100 \mathrm{pb}^{-1}$ and the cross-sections predicted by STARLIGHT, the three $\Upsilon$ resonances are clearly visible above the continuum. 


\section{The High Precision Spectrometer project}

In longer term, the CMS collaboration is studying possibility of the installation of proton spectrometers at $\pm 240 \mathrm{~m}$ and $\pm 420 \mathrm{~m}$ of the IP to measure the very forward protons in association with the system produced centrally, with an acceptance in the $0.002<E_{\text {loss }} / E_{p}<0.12$ region. Effectively, one will use the stronger deflection of the scattered protons by the LHC magnets due to the energy loss $E_{\text {loss }}[10]$.

Dedicated forward stations would be equipped with silicon tracking detectors aiming to reconstruct the proton energy loss with high precision. In order to achieve a resolution of $2 \mathrm{GeV}$ on the mass of the exclusive system, $\mathrm{a} \sim 10 \mu \mathrm{m}$ spatial and $\sim 1 \mu \mathrm{rad}$ angular resolutions are needed. To fulfill these requirements, 3D silicon technology will be used. In addition, forward stations would need to include fast timing detectors with $10 \mathrm{ps}$ resolution in order to reduce the accidental coincidence background from multiple interactions in the same bunch crossing [11].

The future installation of High Precision Spectrometer as a sub-detector of CMS will allow for complementary and unique measurements at the LHC. In particular, one should highlight possibility of detection of the central exclusive production of the Higgs boson, in which the reconstruction of forward protons will allow for determination of $M_{H}$ on event-by-event basis [11]. Similarly, two-photon exclusive production of SUSY pairs could lead to mass reconstruction of the LSP and charged sleptons, with a few $\mathrm{GeV}$ resolution [12]. Finally, such measurements may reveal the existence of new phenomena in high energy photon interactions [13].

\section{References}

[1] J. Ohnemus, T.F. Walsh, and P.M. Zerwas, Phys.Lett.B328 369-373, 1994.

[2] N. Schul, K. Piotrzkowski, Nucl.Phys.Proc.Suppl.179-180:289-297, 2008.

[3] V. Khoze, A. Martin, M.G. Ryskin, Eur.Phys.J.C23:311-327, 2002.

[4] M.G. Albrow, T.D. Coughlin, J.R. Forshaw, Progress in Particle and Nuclear Physics (to be published), arXiv:1006.1289

[5] CMS collaboration, CMS PAS DIF-07-001.

[6] CMS collaboration, JINST 0803:S08004, 2008.

[7] J. Vermaseren, Nucl.Phys.B229 347, 1983.

[8] Nystrand, S. Klein, Phys.Rev.Lett.92 142003, 2004.

[9] H1 collaboration, Phys.Lett.B483 23-35, 2000. Zeus collaboration, Phys.Lett.B680 4-12, 2009.

[10] K. Piotrzkowki, "High Precision Spectrometer Project in CMS", invited talk at the Trento workshop, January 2010

[11] The FP420 collaboration, JINST 4:T10001, 2009.

[12] K. Piotrzkowski, N. Schul, AIP Conf.Proc.1200:434-437, 2010.

[13] J. de Favereau et al., arXiv:0908.2020 